\title{
Analysis of Emergency Task Planning System in Multi-subject Coalitions
}

\author{
Enyan Wang ${ }^{1}$ and Xiangyang $\mathrm{Li}^{2}$ \\ ${ }^{1,3}$ School of Management, Harbin Institute of Technology, Harbin,China. \\ wey0103@126.com
}

\begin{abstract}
Emergency response is a complicated process that requires substantial knowledge, information support and multi-subject collaboration. This paper focuses on the analysis and design of a system for emergency responders in charge of task planning, coordinating and controlling the actions in crisis response. Based on system science theory, we analysed the characteristics of emergency decision-making in times of disaster and discussed the process and internal architecture of the resulting system for developing an emergency plan. We also provide a basic analysis framework for multi-layer, multisubject and multi-task emergency planning in times of disaster. We designed the structure and process of an emergency planning system. Consistent data standards are critical for such a system, and many analyses of crisis response reveal that the lack of such standards hinders efficient critical information flow among incident responders. Therefore, using a team collaborative perspective for emergency planning, we developed an emergency information and plan model for general emergency planning based on the hazard emergency domain and plan ontology concepts. This is a basic utilitarian model for the hierarchical collaborative emergency plan process. We also developed a prototype system and tested its validity for multi-subject task planning.
\end{abstract}

Keywords: Disaster, Emergency Plan, Ontology, Collaborative Planning

\section{Introduction}

Disasters seem to be occurring with increasing frequency and severity, as seen in the Wenchuan Earthquake in China in 2008 and in the Great East Japan Earthquake in March 2011, which triggered a tsunami and ultimately nuclear meltdowns at a nuclear power plant in Fukushima. These types of disastrous events occur suddenly and require timely emergency response, which challenges not only the economic and social system's capability to withstand the disasters but also the emergency decision-making level of the rescue organizations [1]. Emergency decision-making during a disaster therefore plays out under highly uncertain and dynamic conditions. Any mistake during this process can significantly affect the economic development, social stability and ecological health of the region [2]. It is fundamental to prepare structured and coordinated management and to minimize the consequences of potentially ensuing emergencies [3].

In general, emergency response management encompasses a variety of activities, including preparation, early signal detection, planning, task distribution, execution and supervisory control. Construction of a scientific emergency task planning system during a disaster is crucial for building a complete and effective emergency management system [4]. In the emergency response process, an emergency planning system should be able to meet the real-time process requirements, such as the rapid generation, acquisition, analysis, communication and dynamic updating of the activity plan for the various tasks [5]. Additionally, emergency rescue activities rely on team collaboration. An effective emergency plan also depends on the collaboration between different departments from different areas because the collaborative process covers different domains[6]. Hence, the 
system needs to solve the problem of understanding the consistency required by the different domains. System data interoperability is usually seen as crucial for interorganisational communications among various response agencies (e.g., local, state and federal) [7]. Those response agencies use systems independently operated and managed are not interoperable lead to response agencies cannot share task-critical information in a timely manner [8]. In the area of emergency response, many studies have been conducted on how to strength information communications effectively. Solutions need to be based on social media, development of emergency information systems and decision-support systems, which aim at improving information exchange and sharing in complex and uncertain situations. Chen developed a data model usable in response to fire-related extreme events to resolve the issue of consistent data standards for current emergency management practice [9]. These methods are based on information processing technology and various types of human-computer systems applied in specific emergency situations. However, for large-scale disasters, with multiple subjects participating in the emergencyresponse process, a superior command layer has great difficulty maintaining complete control at the field level. There is inadequate study on how to use the information for collaborative planning to reduce ineffective tasks and conflict.

This paper seeks to describe a solution for a multi-subject collaborative planning system and provides the basis for rapid response surrounding inexact and complicated tasks. It first analyses the hierarchy of a multi-subject emergency plan and classifies the types of emergency decision tasks based on emergency management practice. Then, according to the characteristics of a multi-subject emergency activity, we built a general function framework for an emergency task planning system based on a review of existing literature and the theory of a teamwork mechanism. The key point in the research was converted from single-subject behaviour in emergency planning to team-collaboration behaviour. The paper proposes an ontology-based task-planning representation to provide a feasible solution for the inconsistency that exists between multi-subject and multidomain emergency entities. Finally, we developed a prototype system based on the above analysis and report the main evaluation outcomes.

\section{Multi-layer Division in the Emergency Decision}

During integrated planning, decision tasks faced by agents generally differ from one another. In the theory and practice of organization management, decisionmaking is usually divided into three levels - the task layer, the resource layer and the operation layer-in most emergency management systems. This hierarchical arrangement is a common practice in military models of command and control [1011]. The task layer is generally called the strategy layer for explaining the goal of making the plan through an abstract description of the issues at a higher level. This layer also indicates the tasks required to achieve the goal. Additionally, the task layer generates a group of subtask goals, though it does not elaborate on these goals [12]. The main activity in this layer is to analyze the relevant global information and define the direction of the tasks. For example, in an earthquake, the emergency rescue control centre is given the tasks of rescuing the trapped, helping the injured and minimizing damage within the disaster area.

The resource layer refines the tasks from the task layer by analyzing the knowledge and information in the operational environment in detail to provide the resources that are needed for the various tasks and to manage and coordinate these resources [13]. Therefore, the resource layer decision influences the organizational activities to a certain extent. We define the resource types, task constraint conditions, and relevant relationships used for accomplishment of a task by refining the task goals. The collaboration between the resources indicates the task execution process. For example, assume the goal of a task is to avoid leakage of nuclear 
material to another area. In the corresponding resource layer, the fire department is responsible for executing analysis of the resources and division of the goal, according to the expertise of a nuclear leakage professional, and other practical information,

The operation layer, also known as the on-site layer, has the function of conducting the predefined action plan by a single responsibility unit, and will respond flexibly depending on the situation. The judgments, responsiveness and operational speed of the execution subject directly influence the rescue results. Table 1 summarizes the features of the three levels.

Table 1. Emergency Decision Layers

\begin{tabular}{|c|c|c|c|}
\hline & Task Layer & Resource Layer & Operation Layer \\
\hline Information Input & $\begin{array}{l}\text { General complicated } \\
\text { abstract task }\end{array}$ & $\begin{array}{l}\text { Execution activity } \\
\text { plan and constraints }\end{array}$ & $\begin{array}{l}\text { Activity tasks and } \\
\text { requirements }\end{array}$ \\
\hline Information Output & Execution activity plan & $\begin{array}{l}\text { Requirements of } \\
\text { activity tasks }\end{array}$ & $\begin{array}{l}\text { Indecomposable meta- } \\
\text { activities }\end{array}$ \\
\hline $\begin{array}{l}\text { Required } \\
\text { Knowledge }\end{array}$ & $\begin{array}{l}\text { Global information, } \\
\text { various types of models }\end{array}$ & $\begin{array}{l}\text { Specific operational } \\
\text { environment, resource } \\
\text { information, } \\
\text { distribution model }\end{array}$ & $\begin{array}{l}\text { Specific activity } \\
\text { information, } \\
\text { operational rules and } \\
\text { models }\end{array}$ \\
\hline Operation Process & $\begin{array}{l}\text { Issue analysis, direction } \\
\text { definition, task priority } \\
\text { definition }\end{array}$ & $\begin{array}{l}\text { Resource distribution } \\
\text { and equilibrium }\end{array}$ & $\begin{array}{l}\text { Execution, feedback, } \\
\text { information share }\end{array}$ \\
\hline
\end{tabular}

\section{Analytic Framework of Hierarchical, Collaborative Emergency Plan System}

Based on the abovementioned hierarchical division, an emergency plan is a multi-layer and multi-party distributed planning process that involves different emergency activity subjects that collaborate with each other to achieve the goal [14]. The entire process requires continuous information exchange. Therefore, this paper adopted a team collaborative theory to analyze the complete task generation process and build a collaborative planning system.

In teamwork research, the complexity of problems associated with coalitions, such as disaster relief operations, requires that the planning and execution activities of coalition members are performed collaboratively. Such activities cannot consist merely of simultaneous and coordinated individual actions, but the coalition must be aware of and care about the status of the group effort as a whole [15]. Through many different practices we conclude that a common goal is insufficient for guaranteeing that collaborative problem solving will follow. Team members also need to agree on a common solution for achieving their goals. We therefore set forth the following design proposal:

Proposition 1: A multi-subject emergency planning team needs to jointly identify and acknowledge the emergency activity goals and the plans for achieving goals.

The Joint Intention Theory specifies that a group of agents must cooperate by sharing certain mental states about joint activities [15]. The principal idea is that the team's joint activities do not consist merely of coordinated individual actions, but rather of each member's needs.

For efficient collaboration, each team needs to have mutual beliefs about the goals and actions to be performed and the capabilities, intentions and commitments of the other participants. Mechanisms are also needed that enable agents to incorporate planning and acting, avoiding adoption of conflicting intentions and keeping the focus on the information that they actually need for their activities [16].

Proposition 2: All team participants must share the plan in the execution and implementation process. 
Each activity appears as a subtask and is authorized to be a set of constraint mechanisms. When the activity does not satisfy a constraint, a delegation failure would be reported, and the feedback sent to the upper-level plan to trigger a new planning process. In addition, we should also consider the qualitative or quantitative time as a reference to confirm the relationships, such as the sequence or simultaneity, between activities. During the implementation, each subject should continuously exchange the current status and execution progress. Hence, the system can coordinate the activities according to the unified plan goal.

Proposition 3: Team members promise to provide necessary support for other members' activities.

The team collaborative model must be underscored by the idea of mutual support, providing ways for useful information-sharing mechanisms and creation of supportive activities. The principal idea behind mutual support is to enable one agent to have knowledge of the needs of other agents. For example, an agent knows that a specific road is clear so they use this constraint in their plan. However, because the world is dynamic, the road may become blocked. If any other agent finds out that the road is no longer clear they must inform the first agent of this.

An important aspect of team theory is that during the process of task decomposition, team members are not only required to know that a plan exists to enable one or more teammates to perform activities, but also the details of such plan. The agents selected to perform a given sub-activity need to know all the related activities.

\section{Main Function and Process Analysis}

The system should receive real-time data, which is transmitted by different information sources. Therefore, the entire emergency response community is able to quickly confirm the critical attributes, such as the exact location, disaster site situation, basic geographical environment within the range of influence and the progress of the disaster relief. It is vital to maximise sharing of the disaster's overall information by all participants involved in an emergency, partially the related but not in task domain information. It is significant in integrated planning to share such information, rather than just within the work-related and task domain. According to the above analysis, the integrated planning system of emergency decision-making based on the above framework structure has the following functions:

Function 1: Task segmentation and distribution - Based on the results from the situation evaluation and the integration of the global information view, the system generates different tasks. Each complete task is divided into several subtasks. The system also distributes the subtasks to the different emergency plan units according to the task types.

Function 2: Unit plan results generation - According to the distributed tasks, each emergency unit collects the domain knowledge needed for the planning process from the emergency knowledgebase and can quickly generate the emergency plan. The system will also receive the detailed emergency program and activity information from other units to assist with the collaborative emergency activity.

Function 3: Plan fusion and distribution - A globally coherent and non-redundant plan is integrated by eliminating any redundancy and conflicts between the individual plans to obtain a multi-subject collaborative emergency plan. The system also segments the plans into executable nodes (operation layer) according to the task layers. The constraint mechanism should check the activity execution conditions to eliminate any conflicts and then perform a distribution of the standard executive programs.

Function 4: Check the plan status command configuration - The department's plan can be checked in terms of the progress of the activities in the plan, including whether they can be approved. It can make rescuers check the interrelated activities of other 
departments. A visual communication system should also be constructed to visualize all layers of the command architecture. The emergency command, which should be configured at both fixed and mobile terminals, can work alone or via a network to penetrate the forefront of the disaster.

The basic task of the planning system is to provide a mutual operational notation model for emergency task sharing that allows the team members to understand each other's emergency plans and to support each other's emergency activities [17-18]. The emergency domain knowledgebase should be constructed such that it meets the emergency plan requirements. The concept of ontology is introduced to systematically model, acquire and organize the planning domain knowledge. We should also attempt to ensure that the content and notation capacity of the knowledgebase meets the planning process requirements and supports the planning system's solving process to improve the efficiency of the system.

The emergency knowledge is shared and reused through the unified modelling of the emergency planning domain knowledge and construction of an ontology-based emergency task notation model, storage and organization system. The ontology knowledge base includes the multi-source emergency domain objects and their attributes and the operational rules for various types of emergency activities. This knowledge base thus supports key nodes of the intelligent planning process for identification of emergency solutions in the emergency response process.

\section{General Model Construction of an Emergency Plan}

\subsection{Layer Architecture of Emergency Plan Ontology}

The concept of ontology stems from philosophy. However, with the continuous development of computer science, ontology has developed a unique meaning: modal and explicit specification of shared and conceptualised information. The main objective of ontology design is to allow knowledge to be shared and reused and to make this knowledge consistent and uniform by using the same vocabulary, which is based on an agreed contract. In addition, the planning issue requires a standard planning process. In single decision ontology, we only need to consider the efficiency of the decision-making and execution processes. However, in multisubject planning process design, we must consider the common understanding between different subjects. Ontology provides a conceptual shared model that can be used for the construction of a framework that supports the planning and execution processes using unified collaborative planning. In addition, the emergency plan is the result of emergency in the planning process and the collection of a set of activity sequences. This paper defined the ontology notations of a plan that provided the methods of domain information sharing in a multi-layer, multisubject unified emergency plan.

Some approaches exist for construction of an ontology model. $\langle\mathrm{I}-\mathrm{N}-\mathrm{C}-\mathrm{A}\rangle$ (Issues -Nodes-Constraints-Annotations) is a general ontology model that can be used to construct a hierarchically organised multi-subject plan [19]. For universality and practicability, this paper considers: a generalised plan ontology model + an emergency domain ontology model $=$ emergency plan ontology. The hierarchy between the different ontology concepts in the pre-plan can not only improve the efficiency of the ontology construction but also easily extend and integrate the ontology. Table 2 shows the basic hierarchical architecture of the ontology of the emergency plan. 
Table 2. Architecture of Emergency Plan Ontology

\begin{tabular}{|c|c|c|c|}
\hline \multicolumn{3}{|l|}{ Hierarchy } & Relevant Concepts \\
\hline \multicolumn{3}{|c|}{ Top level ontology } & $\begin{array}{l}\text { Emergency plan, emergency planning issues, } \\
\text { emergency activity and method, activity } \\
\text { constraints, event status, emergency objects and } \\
\text { resources, standard activity program }\end{array}$ \\
\hline \multirow{3}{*}{$\begin{array}{l}\text { Middle level } \\
\text { ontology }\end{array}$} & \multicolumn{2}{|c|}{ Shared ontological elements } & Time points, entity variables, sequence \\
\hline & \multirow{2}{*}{$\begin{array}{l}\text { Domain } \\
\text { level } \\
\text { ontology }\end{array}$} & $\begin{array}{l}\text { Plan domain } \\
\text { ontology }\end{array}$ & $\begin{array}{l}\text { Plan, planning issues, constraints, domain } \\
\text { model }\end{array}$ \\
\hline & & $\begin{array}{l}\text { Emergency } \\
\text { domain } \\
\text { ontology }\end{array}$ & $\begin{array}{l}\text { Incident, crisis, emergency entity, resources, } \\
\text { emergency activities }\end{array}$ \\
\hline \multicolumn{3}{|c|}{ Meta-ontology } & Ontology, relationships, roles \\
\hline
\end{tabular}

\subsection{Formalised Model for the Emergency Plan Ontology}

Relying on the concept extraction, this process acquires the ontology concepts and the relationship between the concepts that are necessary to express the emergency plan. The next work is to define the factors involving the practice modelling, including the practical forms of emergency operation, the premise and effect of performance, defining the domain constraints of the action parameters data range, and axiom knowledge involved in the emergency plan creation.

1. Emergency event status: Evs is the status collection of possible emergency events. We also defined evs as a limited and unified proposition collection. Moreover, evs represents the ontology behaviour basis of the plan system such that evs $\in E v S . T$ is the possible time collection such that $t_{i} \in T$.Therefore, $S=\left\{\left(e v s_{1}, t_{1}\right),\left(e v s_{2}, t_{2}\right), \ldots,\left(e v s_{n}, t_{n}\right)\right\}$ represents a set of event sequences, where $\left(e v s_{i}, t_{i}\right)$ is a status description.

2. Emergency plan issues: Depending on the emergency status, the system is required to solve the issue or task description. The planning issues are given after the disaster situation evaluation, and are also known as the primary task s that need solving. Definitions for issues should be based on analysis of the specific circumstances, according to the time characteristics, and return different results. These issues can be divided into operating tasks and model tasks. Operating tasks can be given an initial state and explicit goal state, and need to be actually executed in a specific scenario. The solution of the issue requires time; i.e., in connection with a time interval, and the returned result is some sort of event status, such as flood control and embankment, or transport of materials. Model tasks do not need to be completed in a particular context. They are often called the existing algorithms performed by machines. Because computation time is limited, they cannot consider the execution time of the problem, such as path planning, the shortest path problem, and the implementation of results is some form of numerical data. The expressions of the two types of issues are different. The description operation tasks should include the initial status, goal status, class, level and relevant instruction.

3. Emergency resources: These are classified as activity, consumable and application resources. Activity resources are the activity execution subjects, such as the emergency command centre, fire brigade and medical staff. Consumable resources include energy, such as water and electricity, and consumable equipment. Application resources include other resources such as ambulances and fire trucks. An emergency resource is represented as follows: (resource name, class, subject, relevant attribute description: attribute, attribute value). 
4. Emergency response method: This is an act or operation, the smallest unit of behaviour. It is directly executed by a single emergency organisation entity. It is a complete execution logic unit and inseparably includes the action, conditions and effect. To build a unified model, five tuples are available: EmergencyResponse $=($ Name, Participant, Parameter [Inputs, Outputs], Result, Constraints), where "Name" represents a name of the basic emergency response unit. This should be a specific activity, such as "Transport", and also a method, such as the address format conversion (AddressConversions). "Parameter" represents the parameters required to perform activities or a method. The two important kinds of parameters are the input and output parameters. "Inputs" represent the input information in which the basic unit execution is required, such as "Transport", and the formal description framework is "from (? X)" "to (? Y)". Then inputs are variables $\mathrm{X}$ and $\mathrm{Y}$, reflecting the starting and ending location, which should be defined before the activity is executed. "Outputs" represent the results of activities or methods that are a particular parameter.

5. Execute result: The effect of a method or operator is expected. The results can be divided into: changing the event state and completing an operation. The results of an emergency response method can be divided into status and effect.

6. Standard emergency operating procedures: These are normalised emergency operations; a pre-defined set of activities executable by the emergency rescue agents. This set of activities is a set of emergency response disposition activities, such as transport procedures of relief goods, treatment procedures of flood control dams, victim transport and resettlement programs. A standard emergency program is associated with the response process of certain disaster events. It is composed of two parts: methods set for emergency response and operation order (which is divided into, for example, sequence, split or choice).

7. Activity constraints: These include the limitations of the activity itself, the resources and the relationships among different activities. They can be classified as key and subsidiary constraints, represented as: (constraint type, constraint relationship, attribute condition in the description framework).

1) Environmental constraints: These concern the environment at the emergency site, such as the weather and the event status. They can be modally represented as: <Type _cons: world-state, relationship: condition> ((attribute object [attributequalifies]), value) attributes or attribute description. The value type can be defined as string, variable, activity, issue, number or symbol.

2) Time constraints: These indicate the time required for execution of each activity. Each activity in the emergency plan should be based on the exact time axis, which can represent the activity relationship and the necessary activity execution times through either qualitative or quantitative time parameters. These constraints can be represented as: <Type_cons: temporal, relation: interval> intervals: (Act_id,(t_initial, t_final)) or temporal-relation: (Act_id-x, Act_id-y).

3) Emergency resource constraints: These indicate the resources that should be used for execution of a specific activity and can be represented as: <Type_cons: resource, relationship: resource-type> ((resource object [resource-qualifier] [resource-range]), value).

4) Delegation constraints: These can be represented as: <Type_cons: commitment, relationship: commit-type> ((Act_id-i,agent-j),true/false). We can also define additional constraints, such as preference, conflict, execution text and permission constraints.

8. Goal description: The emergency plan unit goal is described to attain all of the event statuses of the planned goals in the emergency status collection. 
9. Emergency plan: This is represented as: $\operatorname{Plan}(O, P)=\bigcup_{i=1}^{n} p \operatorname{plan}\left(o_{i}, p_{i}\right)$, where $p_{i}=(G D, P I$, Actsets $), G D$ is the goal description, $P I$ is the plan issue, and Actsets is an activity description in the plan or the ordered activity meta set.

10. Annotation information: This information is used to explain the information that is not easy to indicate in other parts of the framework, including the manually controlled decision information. An example includes the addition or revision of certain decision information or basic planning information.

The relationships between the concepts of the emergency plan $E P$ _ Relations represent the two-tuples collections between the emergency plan concepts. The key relations are as follows:

1) Paradigmatic relationships (composes) indicate that an emergency concept entity is composed of several other concept entities.

2) Inheritance relationships (inherits) indicate that one of two emergency entities is part of a subclass and that the other forms part of the parent class such that the subclass inherits all the attributes of its parent class. For example, the resource constraints are a subclass of constraints.

3) One-way dependencies (implements) indicate that a subject and an activity of the emergency plan have an active and passive relationship, such as a subject plan and an event execution.

4) Time sequences (follows) indicate the time relationship between two activities, such as input constraints, output constraints and time range constraints.

5) Cause-and-effect relationships (affects) indicate a direct relationship between two actions by the resources required such that one activity generates another activity. For example, a cause-and-effect relationship, which is also a special time sequence relationship, is the combination of an input constraint and an output constraint.

Figure 1 shows the relationships among emergency plan ontology concepts.

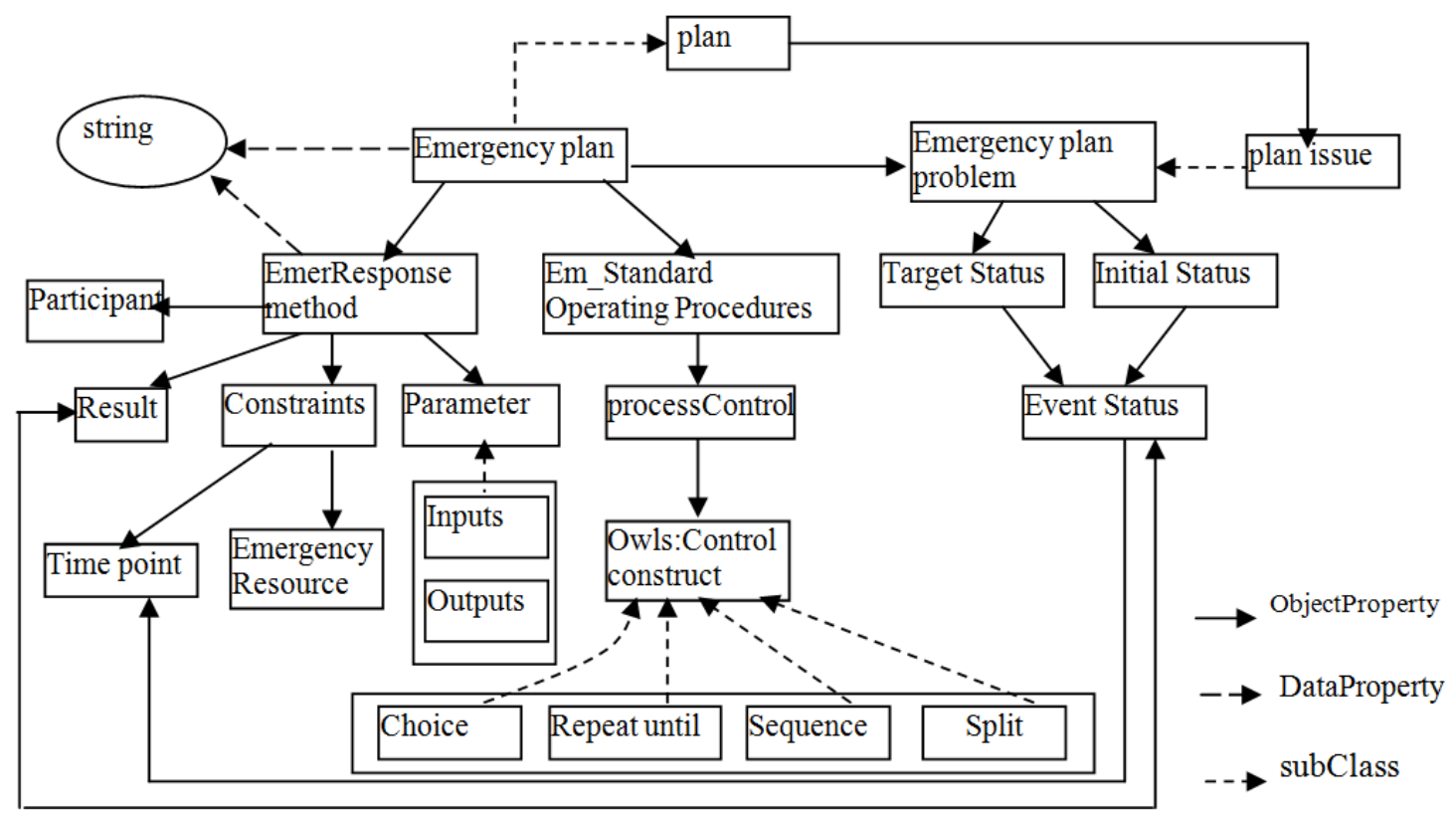

Figure 1. Description of Relationships among Emergency Plan Ontology Concepts 


\section{Prototype System Test}

Based on the analyses in the previous sections, we built an Internet-based prototype system. After the prototype development, we simulated different organizations' planning processes in earthquake disasters by using the prototype system to test its quality of user interaction and on the capability of real supporting collaboration planning in the emergency response. Figures 2 and 3 provide a snapshot of the prototype system. The data stored on the Internet consist of a set of MySQL relational databases and could be accessed by computers with Internet access. That authenticated organization or personnel with an approved user name and password could access the system.

The system's functions include incident management, department management, emergency response activities management and emergency plan management. A common basic structure was replicated in all user pages to ensure consistency and support ease of use. A generic page includes the following fundamental elements, highlighted in Figure 3, which shows the page of the police office: (1) page header with logo, (2) "signed in as" area, with the username and time of the user logged in, (3) main menu bar, which is different for each user role, (4) function list in the page and (5) main area, which depends on user role and system state; for example Figures 2 and 3 show the police office making plans and querying its own plans and related departments' plans. It can show the relationship between the tasks of their own departments with other tasks and the schedule of response activities.

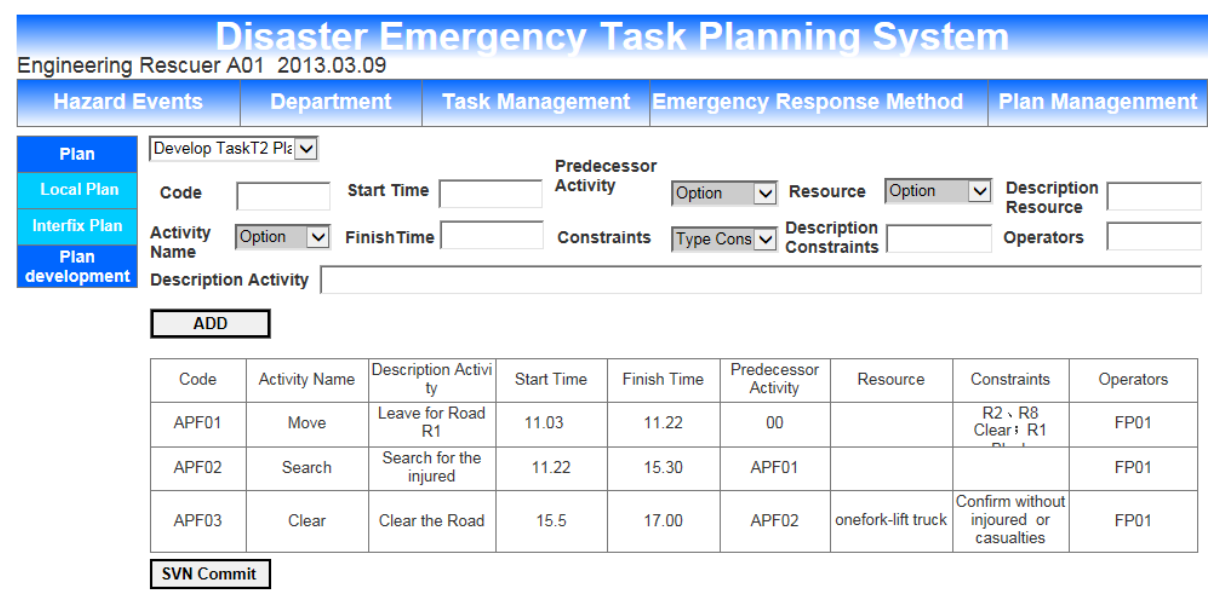

Figure 2. Plan Generation Interface

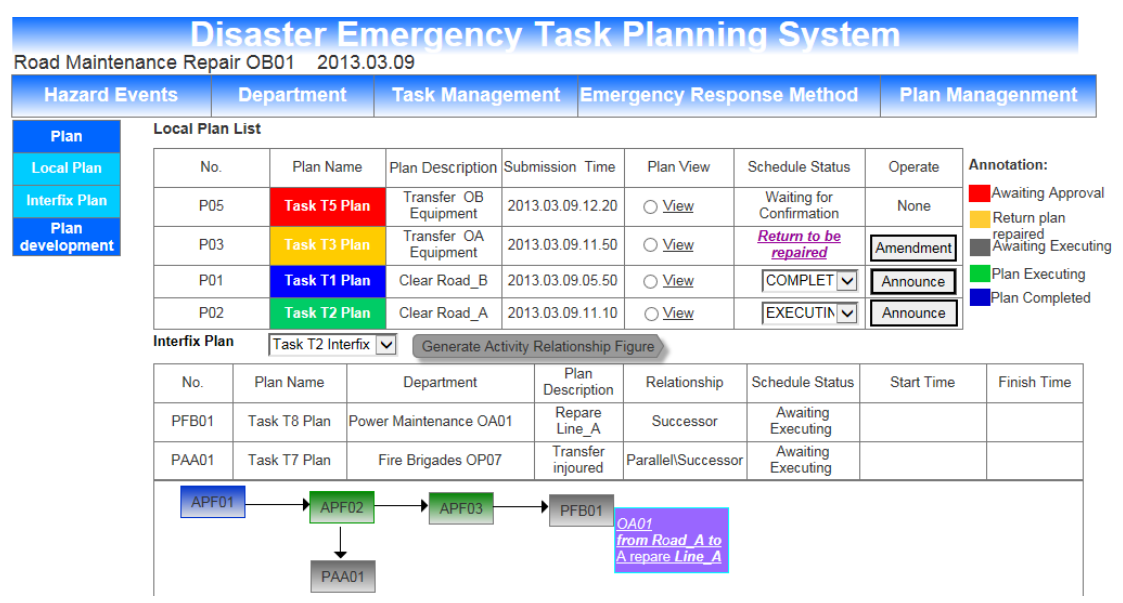

Figure 3. Plan Checking Interface 
The following methodology, purposely designed for the case at hand, was adopted to perform assessment of collaboration effectiveness:

1. An evaluation team was first defined, including 16 participants from local fire and police departments and hospitals, holding an average of five-plus years of work experience, and with experience as a participant in hazard rescue in some capacity. The emergency team created for experimentation included one command centre (strategic level), one police station, ambulance centre and fire station (resource layer), two ambulance teams (AT), whose function is to rescue buried civilians; two fire brigades (FB), whose function is to extinguish fires; and two police forces (PF), whose function is to clear roads (operation layer); separate working rooms have been prepared to simulate a distributed setting.

2. A mock emergency scenario was created. Suppose an earthquake occurs in the region, leading to building collapses, traffic congestion and fires in some areas. Evaluators assume response roles such as command centre, fire fighters, police, and ambulances, and based on the information to plan.

3. All users, based on their use of the prototype system, gave a score on whether it could help in conducting planning. We adopted a general scoring method [20]. Users were instructed to give their opinion on the test case through a qualitative value in the ordered set \{ unacceptable, partially acceptable, adequate, satisfactory, completely satisfactory $\}$ according to the degree to which the system functions meet their expected requirements. These values were then mapped onto the integer scale $\{1,2,3,4,5\}$, where an average $\geqslant 4$ meant the system was excellent, $\geqslant 3$ meant was acceptable, and otherwise the system cannot support the planning process.

The final assessment of the DSS provided the result 3.87. In addition to the evaluation score, informal opinions were gathered from the users during the assessment activities.

All users judged the system as useful for task management of emergency situations. Users in the operation layer thought the system could provide the involved teams and agents with clear insight into the roles and functions they would undertake in the overall rescue; thus understanding the action relationship between their team and others. The agents were satisfied with this function, and thought it could improve understanding between different groups to reduce discrepancies and conflicts. Users in the operation layer also thought the system could provide the involved teams and agents with clear insight into the roles and functions they would undertake in the overall rescue; understanding the action relationship between their team and others. This aspect of the system could also appeal to the agents, and likewise improve the understanding between different groups to reduce discrepancies and conflicts. The majority of the members of the emergency team agreed that the DSS makes them more effective and focused on the goal. The task layer can provide strong improvements in task allocation and supervision, and reducing task conflicts and inappropriate tasks. Users pointed out that the system can prevent different people from simultaneously making illogical or even contradictory decisions. The possibility of monitoring the execution of plans in a simple and structured way was particularly appreciated.

\section{Conclusion}

Based on the urgent requirements of an emergency plan during a disaster, this paper introduced a teamwork mechanism for a multi-subject collaborative plan. Herein, we also proposed a structure framework for the emergency plan system based on the ontology model. The framework comprehensively uses the domain knowledge in an emergency ontology knowledge base to plan and solve the emergency domain issue. We also 
introduced a formalized description of construction of an emergency domain plan ontology notation to support the system's operation.

The prototype system can dynamically plan tasks and generate a global optimized emergency task solution based on the different stages and units of the emergency plan. The segmentation, formulation and global integration of the plan would be executed through the superior-subordinate relationship and decision-making levels between departments. It makes the agencies involved in the emergency response share their plans and status with each other, and obtains an overall perspective of the disaster rescue. This framework design lets us flexibly address different types of emergency management problems. By providing a more professional, intelligent and humanised decision process to support the planning function, the system can also improve information sharing to assist emergency personnel in solving the complicated domain plan issue.

\section{References}

[1] W. C. Fan, "Advisement and Suggestion to Scientific Problems of Emergency Management for Public Incidents", China Science Foundation, vol. 2, (2007), pp. 71-76.

[2] D. E. Smith, "Choosing Objectives in Oversubscription Planning", The 14th International Conference on Automated Planning and Scheduling, (2004).

[3] D. Fogli and G. Guida, "Knowledge-centered design of decision support systems for emergency management”, Decision Support Systems, vol. 55, (2013), pp. 336-347.

[4] J. C. Pine, "Technology in emergency management', John Wiley \& Sons, Washington DC, (2007).

[5] S. Jain and C. R. McLean, "An integrating framework for modeling and simulation for incident management", Journal of Homeland Security and Emergency Management, vol. 3, no. 1, (2006).

[6] D. Mendonça and W. A. Wallace, "A Cognitive Model of Improvisation in Emergency Management", IEEE Transactions on Systems, Man, and Cybernetics-Part A (Systems \&Humans), vol. 37, no. 4, (2009), pp. 547-561.

[7] F. F. Townsend, "The Federal Response to Hurricane Katrina Lessons Learned", The White House, Washington, DC, (2006).

[8] R. Chen, R. Sharman, H. R. Rao and S. J. Upadhyaya, "Coordination in Emergency Response Management”, Communications of the ACM, vol. 51, no. 5, (2008), pp. 66-73.

[9] R. Chen, R. Sharman, R. H. Rao and S. J. Upadhyaya, "Data Model Development for Fire Related Extreme Events: An Activity Theory Approach”, MIS Quarterly, vol. 37, no. 1, (2013), pp. 125-147.

[10] T. Killion, "Decision Making and the Levels of War", Military Review, vol. 80, no. 6, (2000), pp. 66-70.

[11] B. Ferguson, "Foundations of New Zealand Military Doctrine", Headquarters New Zealand Defence Force Publication, (2004).

[12] C. A. Siebra, "A Unified Approach to Planning Support in Hierarchical Coalitions", University of Edinburgh Press, UK (2006).

[13] L. Castillo, J. Fdez-Olivares and O. Garc Ma-Perez, "Efficiently Handling Temporal Knowledge in an HTN Planner", Proceedings of International Conference on Automated Planning and Scheduling (ICAPS-06), Cumbria, UK, (2006), pp. 63-72.

[14] M. Asuncion, L. Castillo and J. Fdez-Olivares, "SIADEX: An Integrated Planning Framework for Crisis Action Planning", AI Communications, vol. 18, no. 4, (2005), pp. 257-268.

[15] J. Levesque, P. Cohen, and J. Nunes, "On Acting Together", Proceedings of the Eighth National Conference on Artificial Intelligence, Los Altos, California, USA, (1990), pp. 94-99.

[16] B. Grosz, L. Hunsberger and S. Kraus, "Planning and Acting Together", AI Magazine, vol. 5, no. 2, (1999), pp. 23-34.

[17] M. Ghallab, D. Nau and P. Traverso, "Automated Planning: Theory and Practice", Elsevier Press, (2004).

[18] Y. Xi, C. G. Cao and A. M. Bai, "Research and Application of Ontology-based Intelligent Planning Method", Computer Science, vol. 38, no. 2, (2011), pp. 175-178.

[19] A. Tate, " $\langle$ I-N-C-A $>$ : An Ontology for Mixed-Initiative Synthesis Tasks", Proceedings of the IJCAI Workshop on Mixed-Initiative Intelligent Systems,Acapulco, Mexico, (2003).

[20] A. M. Lund, "Measuring usability with the USE Questionnaire", STC Usability SIG Newsletter, vol. 8, no. 2, (2001). 


\section{Authors}

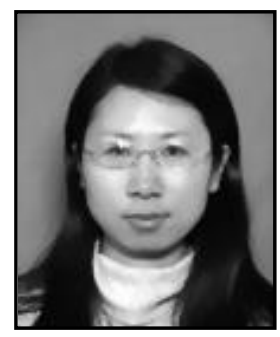

Enyan Wang, lecturer of School of Management, Harbin Institute of Technology (weihai), received her master degree in Management Science and Engineering in 2004. Her main research fields include decision support system.

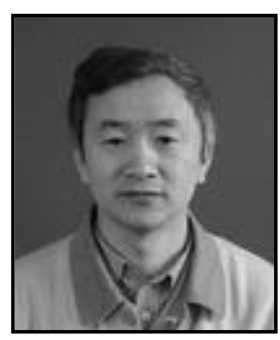

Xiangyang Li, Professor, he is a graduate and doctoral tutor of School of Management, Harbin Institute of Technology. His main research fields include disaster emergency management and management information system. 